\title{
Variational dimension reduction in nonlinear elasticity: a Young measure approach
}

\author{
Lorenzo Freddi ${ }^{1}$ and Roberto Paroni ${ }^{2}$ \\ 1 Dipartimento di Matematica e Informatica, via delle Scienze 206, 33100 Udine, \\ Italy lorenzo.freddi@dimi.uniud.it \\ 2 Dipartimento di Architettura e Pianificazione, Università degli Studi di Sassari, \\ Palazzo del Pou Salit, Piazza Duomo, 07041 Alghero, Italy paroni@uniss.it
}

\begin{abstract}
Starting form 3D elasticity, we deduce the variational limit of the string and of the membrane on the space of one and two-dimensional gradient Young measures, respectively. The physical requirement that the energy becomes infinite when the volume locally vanishes is taken into account in the string model. The rate at which the energy density blows up characterizes the effective domain of the limit energy. The limit problem uniquely determines the energy density of the thin structure.
\end{abstract}

\section{Introduction}

A first variational derivation of the energy of a string starting from the 3D nonlinear elasticity is due to Acerbi, Buttazzo and Percivale [1]. Following the same leading ideas, Le Dret and Raoult [17] derived the energy of a thin film. The integrands involved in the bulk energy by them derived are quasiconvex. More precisely they are the quasi-convex envelope $Q W_{0}$ of a function $W_{0}$ (denoted $f_{0}$ for the string), which is obtained from the 3D free energy density by solving a suitable minimization problem.

It is well known that quasi-convex integrands with appropriate growth conditions lead to the existence of minimizers of the total energy. If we are dealing with martensitic materials, we can not conclude that $Q W_{0}$ is the energy density, since the infimum of the total free energy is not attained, in general. Hence the question: what is the energy density to be considered for the thin structure?

It must be noticed that from the results of Acerbi, Buttazzo and Percivale, or of Le Dret and Raoult as well, we can not deduce that $W_{0}$ is the free energy, since there are an infinite number of functions $Z$ such that $Q Z=Q W_{0}$. It is also well known, in phase transition theory, that it is the free energy that determines the microstructure and not its quasi-convex envelope. Thus the problem at hand is of noticeable importance in applications. 
To explain how we derive the energy density of the thin structure, we must be sligthly more specific. The asymptotic methodology initiated in [1] is the following: a sequence of bodies given in a cylindrical configuration of diameter or thickness $\varepsilon$ is considered. For each of these bodies the total energy is known. Under quite general assumptions on the energy density there are different topologies which ensure compactness to the family of minimizers (or quasi-minimizers) of these energies. Once chosen one of these topologies, the thin structure model is obtained by passing to the limit as $\varepsilon \rightarrow 0$ in an appropriate variational sense ( $\Gamma$-convergence). Roughly speaking, this variational limit ensures the convergence, in such topology, of minimizers of the energy at level $\varepsilon$ to the minimizers of the thin structure problem. The obtained limit problem depends on the chosen topology. As said before, typically the infimum of the total free energy of a martensitic material is not attained. The minimizing sequences shall, in general, develop fine scale oscillations, which, according to the interpretation due to Ball and James [3, 4], model the microstructure experimentally observed in specimens of phase transforming materials. Thus, in phase transforming problems the "main properties" of the minimizing sequences are to be determined. This suggests that when we pass to the limit as $\varepsilon \rightarrow 0$ we should try to use a topology which, loosely speaking, ensures the convergence of the "main properties" of the minimizing sequences at level $\varepsilon$ to the "main properties" of the minimizing sequences of the thin structure problem. We achieve this by embedding the 3D problems into a space of Young measures, see L.C. Young [22], which is one of the most successful tools used to characterize the oscillatory behaviour of sequences of functions. In this way we derive a limit functional which has a feature missing in all the other previously obtained variational limits: it uniquely determines the energy density of the thin structure.

This methodology introduces several difficulties which are completely missing in the work of Acerbi, Buttazzo and Percivale. Like them, we perform the computation of the $\Gamma$-limit under the requirement that the energy becomes infinite when the volume locally vanishes, that is $\lim _{\operatorname{det} F \rightarrow 0^{+}} f(F)=+\infty$. The same requirement is also met in a recent paper of Ben Belgacem [7]. In contrast with $[1,7]$, we need to specify the rate at which the energy blows up when the volume decreases. In fact, in the cited papers, the obtained $\Gamma$-limit involves the convexification of the energy density, which completely disregards the behaviour of the energy near vanishing-volume deformations. On the other hand, in the Young measure setting, where no convexification appears, the growth near such small deformations is as much important as the growth for large deformations. This reflects on the fact that the domain of the limit functional strongly depends on the prescribed growths.

The martensitic thin film model is obtained under the usual growth conditions of order $p$. This problem has been studied previously by Bhattacharya and James [8] who have considered a body characterized not only by a free energy but also by an interfacial energy which, mathematically speaking, behaves as a viscosity term and hence the limit problem does not contain any 
quasi-convex envelope. About one year later, Shu [21] has shown that letting the interfacial energy go to zero the variational limit coincides with that obtained by Le Dret and Raoult. Bělík and Luskin [6] have observed that with the energy considered by Bhattacharya and James [8] the deformations with finite energy cannot have sharp interfaces between compatible variants. For this reason they set the problem within the framework of functions with bounded Hessian and consider an interfacial energy proportional to the square of the total variation of the deformation gradient. Other interesting results on the Young measure theory of thin films have also been obtained, from a slightly different point of view, by Bocea and Fonseca [9].

The paper is written in a quite concise manner and all technical details, for which we refer to Freddi and Paroni $[12,13]$, have been left out.

\section{The $\Gamma$-convergence tool}

The energies of the thin structures are obtained by taking a limit in the variational sense of $\Gamma$-convergence. Roughly speaking, the $\Gamma$-limit ensures convergence of minimizers of the energy at level $\varepsilon$ to minimizers of the thin structure problem. In fact, we use a variant of De Giorgi's $\Gamma$-convergence, which has been introduced by Anzellotti, Baldo and Percivale in [2] and allows to treat families of functionals defined on a space which may be different from the domain of the limit. Let us recall here just the definition, referring for a precise formulation of the variational properties to [2] and to the books of Braides [10] and Dal Maso [11]. Let $X$ be a set, let $(Y, \tau)$ be a topological space and let $q: X \rightarrow Y$. Given a sequence $F_{n}: X \rightarrow \overline{\mathbb{R}}$ and a point $y \in Y$, let us denote by

$$
\begin{aligned}
\Gamma(q, \tau Y) \liminf _{n \rightarrow \infty} F_{n}(y) & :=\inf \left\{\liminf _{n \rightarrow \infty} F_{n}\left(x_{n}\right): q\left(x_{n}\right) \stackrel{\tau}{\rightarrow} y\right\}, \\
\Gamma(q, \tau Y) \limsup _{n \rightarrow \infty} F_{n}(y) & :=\inf \left\{\limsup _{n \rightarrow \infty} F_{n}\left(x_{n}\right): q\left(x_{n}\right) \stackrel{\tau}{\rightarrow} y\right\},
\end{aligned}
$$

the $\Gamma$-lower and, respectively, the $\Gamma$-upper limit at the point $y$. If they turn out to be equal and $F(y)$ denotes their common value then we say that the sequence $\Gamma(q, \tau Y)$-converges to $F(y)$ and we write $\Gamma(q, \tau Y) \lim _{n \rightarrow \infty} F_{n}(y)=F(y)$. Given a family $F_{\varepsilon}: X \rightarrow \overline{\mathbb{R}}$ we say that it $\Gamma(q, \tau Y)$-converges to $F: Y \rightarrow \overline{\mathbb{R}}$ at a point $y \in Y$, and we write $\Gamma(q, \tau Y) \lim _{\varepsilon \rightarrow 0} F_{\varepsilon}(y)=F(y)$, if for any sequence $\varepsilon_{n}$ of positive reals converging to 0 we have that $\Gamma(q, \tau Y) \lim _{n \rightarrow \infty} F_{\varepsilon_{n}}(y)=F(y)$.

\section{Young measures}

Let, in the current section, $\Omega$ be an open bounded subset of $\mathbb{R}^{n}$. Let $M\left(\mathbb{R}^{m}\right)=C_{0}\left(\mathbb{R}^{m}\right)^{*}$ denote the space of $\mathbb{R}$-valued Borel measures on $\mathbb{R}^{m}$ 
and $L_{w}^{\infty}\left(\Omega ; M\left(\mathbb{R}^{m}\right)\right)=L^{1}\left(\Omega ; C_{0}\left(\mathbb{R}^{m}\right)\right)^{*}$ the dual of $L^{1}$. An element $\mu \in$ $L_{w}^{\infty}\left(\Omega ; M\left(\mathbb{R}^{m}\right)\right)$ can be viewed as a parametrized measure, that is a map $x \mapsto \mu_{x}$ between $\Omega$ and $M\left(\mathbb{R}^{m}\right)$, which is essentially bounded and weakly* measurable in the sense that the functions $x \mapsto\left\langle\mu_{x}, \varphi\right\rangle$ are measurable for every $\varphi \in C_{0}\left(\mathbb{R}^{m}\right)$. The subscript $w$ in the notation $L_{w}^{\infty}$ refers to this weak* measurability. If $\mu_{x}$ is a probability for a.e. $x \in \Omega$ then $\mu$ is called a Young measure and $\mathcal{Y}\left(\Omega ; \mathbb{R}^{m}\right)$ will denote the space of such Young measures. For instance, if $u: \Omega \rightarrow \mathbb{R}^{m}$ is a measurable function then $\delta_{u(\cdot)} \in \mathcal{Y}\left(\Omega ; \mathbb{R}^{m}\right)$.

The space $L_{w}^{\infty}\left(\Omega ; M\left(\mathbb{R}^{m}\right)\right)$ will be endowed with the weak* convergence induced by the duality with $L^{1}$. Hence $\mu^{n} \rightarrow \mu$ weakly* in $L_{w}^{\infty}\left(\Omega ; M\left(\mathbb{R}^{m}\right)\right)$ iff

$$
\int_{\Omega}<\mu_{x}^{n}, \varphi>g(x) d x \rightarrow \int_{\Omega}<\mu_{x}, \varphi>g(x) d x, \quad \forall \varphi \in C_{0}\left(\mathbb{R}^{m}\right), \forall g \in L^{1}(\Omega)
$$

where $\langle$,$\rangle stays for integration of \varphi$ with respect to the involved measure.

Finally, we say that a sequence of measurable functions $\left(u_{n}\right)$ generates $\mu$ if $\delta_{u_{n}(\cdot)} \rightarrow \mu$ weakly* in $L_{w}^{\infty}\left(\Omega ; M\left(\mathbb{R}^{m}\right)\right)$.

\section{A 3D-1D reduction problem}

Let $\omega$ be an open, bounded subset of $\mathbb{R}^{2}$ and let, for every $\varepsilon>0$,

$$
\Omega_{\varepsilon}=\left\{x=\left(x_{\alpha}, x_{3}\right) \in \mathbb{R}^{2} \times \mathbb{R}: x_{\alpha} \in \varepsilon \omega, x_{3} \in(0, \ell)\right\},
$$

a three dimensional cylinder that we consider as the reference configuration of a hyperelastic body, which reduces to a $1 \mathrm{D}$ region as $\varepsilon$ goes to zero. Our aim is to obtain the energy of an elastic string as limit of the total energy of 3D bodies occupying the regions $\Omega_{\varepsilon}$. Without loss of generality we can assume that $\omega$ contains the origin and that $|\omega|=1$.

The stored energy density $f: \mathbb{R}^{3 \times 3} \rightarrow(-\infty,+\infty]$ in the reference configuration $\Omega_{\varepsilon}$ is assumed to be continuous and to satisfy the following growth assumptions which include the whole class of Antman materials

$$
\begin{aligned}
\operatorname{det} F \leq 0 & \Rightarrow f(F) \equiv+\infty, \\
\operatorname{det} F>0 & \Rightarrow \text { there exists two constants } C \geq c>0 \text { such that } \\
& c\left(\frac{1}{|\operatorname{det} F|^{s}}+|F|^{p}-1\right) \leq f(F) \leq C\left(\frac{1}{|\operatorname{det} F|^{s}}+|F|^{p}+1\right),
\end{aligned}
$$

for suitable $p \in[1,+\infty)$ and $s \in(0,+\infty)$.

The hypothesis that the material is homogeneous, which relays in the assumption that $f$ be independent of the point in the reference configuration, is not essential and can be easily dropped. We refer to [13] for a treatment of the non-homogeneous case where moreover also the diameter of the crosssection is allowed to change from point to point.

Up to the scaling factor $1 / \varepsilon^{2}$, the total energy $I_{\varepsilon}$ of the body is given by 


$$
I_{\varepsilon}(y)=\frac{1}{\varepsilon^{2}} \int_{\Omega_{\varepsilon}} f(D y(x)) d x-\int_{\Omega_{\varepsilon}} \hat{g}^{\varepsilon}(x) \cdot y(x) d x,
$$

where the body force densities $\hat{g}^{\varepsilon}$ are taken in $L^{p^{\prime}}\left(\Omega_{\varepsilon} ; \mathbb{R}^{3}\right)$, with $1 / p+1 / p^{\prime}=1$. Assuming, for instance, the body to be clamped on $\varepsilon \omega \times\{0\}$, the equilibrium configurations will be found by minimizing the energy $I_{\varepsilon}$ over all $y \in W^{1, p}\left(\Omega_{\varepsilon} ; \mathbb{R}^{3}\right)$ such that $y\left(x_{1}, x_{2}, 0\right)=\left(x_{1}, x_{2}, 0\right)$.

\section{Scaling energies and passing to a fixed domain}

The scaling factor $1 / \varepsilon^{2}$ in front of the energy functionals serves to avoid the trivial case where the $\Gamma$-limit is identically zero. The choice of different scaling exponents would provide other limit models with their own physical meaning. For instance, $1 / \varepsilon^{3}$ and $1 / \varepsilon^{4}$ lead to rod theories; these cases has been studied in a quite different setting by Mora and Müller $[18,19]$.

To perform our analysis, it is convenient to put all the energy integrals on the same domain $\Omega:=\Omega_{1}$, which is independent of $\varepsilon$, by the change of variables $x_{\alpha}^{\prime}=\varepsilon x_{\alpha} \alpha=1,2$. This gives to the energy functionals the following form

$$
I_{\varepsilon}^{\Omega}(y):=\int_{\Omega} f\left(\frac{D_{\alpha} y}{\varepsilon} \mid D_{3} y\right) d x-\int_{\Omega} g^{\varepsilon} \cdot y d x,
$$

where $D_{\alpha} y$ denotes the first two columns of the deformation gradient, while $D_{3} y$ is the third column. Hereafter, for simplicity, we assume that the body force densities $g^{\varepsilon}$ do not depend on $\varepsilon$ and set $g:=g^{\varepsilon}$. The total energy $I_{\varepsilon}^{\Omega}$ has to be minimized over all $y \in W^{1, p}\left(\Omega ; \mathbb{R}^{3}\right)$ such that $y\left(x_{1}, x_{2}, 0\right)=\varepsilon\left(x_{1}, x_{2}, 0\right)$.

\section{Previous results and some remarks}

Acerbi, Buttazzo and Percivale [1] studied the problem under the following growth assumptions

$$
\begin{aligned}
& \operatorname{det} F \leq 0 \Rightarrow f(F) \equiv+\infty, \\
& \forall \delta>0 \exists C_{\delta}>0 \text { s.t. } \operatorname{det} F \geq \delta \Rightarrow f(F) \leq C_{\delta}\left(|F|^{p}+1\right), \\
& \exists c>0 \text { s.t. } c\left(|F|^{p}-1\right) \leq f(F),
\end{aligned}
$$

for a suitable $p \in[1,+\infty)$. The $\Gamma$-limit of the functionals $I_{\varepsilon}$ is taken by them under the norm convergence in $L^{p}\left((0, \ell) ; \mathbb{R}^{3}\right)$ of the average of the deformation over the cross section and the resulting $\Gamma\left(L^{p}\right)$-limit is

$$
G_{0}(v)=\int_{0}^{\ell} f_{0}^{* *}\left(v^{\prime}\right) d x_{3}-\int_{0}^{\ell} A v^{\alpha} g \cdot v d x_{3}
$$

if $v \in W^{1, p}\left((0, \ell) ; \mathbb{R}^{3}\right)$ with $v(0)=0 . f_{0}^{* *}$ is the convex envelope of the function

$$
f_{0}(z):=\min \left\{f(\bar{F} \mid z): \bar{F} \in \mathbb{R}^{3 \times 2}\right\}, \quad z \in \mathbb{R}^{3}
$$


and $A v^{\alpha}$ denotes the integral mean value with respect to $x_{1}$ and $x_{2}$.

Under the assumptions (2), conditions (3) are satisfied and a simple computation shows that $f_{0}$ satisfies the following estimates

$$
c\left(\frac{1}{|z|^{q}}+|z|^{p}-1\right) \leq f_{0}(z) \leq C\left(\frac{1}{|z|^{q}}+|z|^{p}+1\right) \text { for every } z \neq 0,
$$

with $q=\frac{p s}{p+2 s}$, for suitable positive constants $C$ and $c$.

In fact, the convexification of the energy density completely disregards the behaviour of the energy near vanishingvolume deformations. On the other hand, in the Young measure setting, where no convexification appears, the growth near such small deformations is as much important as the growth for large deformations.

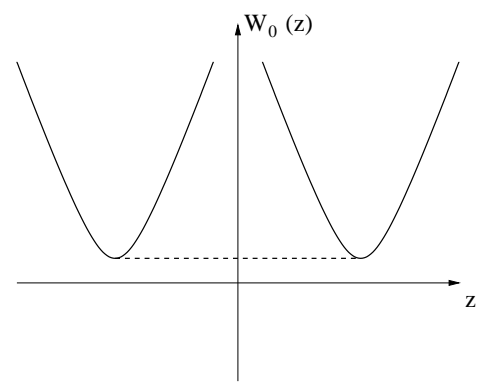

Then we have to expect that conditions (3) be not precise enough to characterize the domain of the limit problem in terms of Young measures. Indeed, we shall see that two different choices of the exponents $s$ and $p$ in (2), will lead to limit problems defined on different spaces.

\section{The Young measure setting}

Acerbi, Buttazzo and Percivale, in taking the $\Gamma$-limit, used the norm topology of $L^{p}$. Nevertheless, there are other topologies which ensure compactness to the minimizing sequences which can choose to compute the $\Gamma$-limit. Limit problems provided by different topologies show different ability in describing the behaviour of the minimizing sequences. As already explained, we shall work within the framework of Young measures.

Via the Dirac mass supported on the gradient, the space of functions $W^{1, p}$ with some prescribed boundary conditions can be identified with a subspace of $L_{w}^{\infty}$. Thus we can extend the functionals $I_{\varepsilon}^{\Omega}$ to $L_{w}^{\infty}\left(\Omega ; M\left(\mathbb{R}^{3 \times 3}\right)\right)$ by setting

$$
I_{\varepsilon}^{\infty}(\mu)=\left\{\begin{array}{cl}
I_{\varepsilon}^{\Omega}(y) & \text { if } \exists y \in \mathcal{A}_{\varepsilon}^{\Omega} \text { s.t. } \mu=\delta_{D y(\cdot)} \\
+\infty & \text { otherwise in } L_{w}^{\infty}\left(\Omega ; M\left(\mathbb{R}^{3 \times 3}\right)\right) .
\end{array}\right.
$$

Due to the growth constraints (2), the effective domain of the limit functional will consist of those Young measures which are characterized by a certain kind of growth, as precised by the following definition.

Definition 1. Let $p \geq 1$ and $q>0$. With $\mathcal{Y}^{-q, p}\left((0, \ell) ; \mathbb{R}^{m}\right), m \in \mathbb{N}$, we denote the set of Young measures $\nu \in \mathcal{Y}\left((0, \ell) ; \mathbb{R}^{m}\right)$ such that

$$
\int_{0}^{\ell} \int_{\mathbb{R}^{m}}\left(|z|^{-q}+|z|^{p}\right) d \nu_{t}(z) d t<+\infty
$$


The Young measures in this spaces can be characterized as those generated by sequences $\left(z_{j}\right)$ such that $\left(\left|z_{j}\right|^{-q}+\left|z_{j}\right|^{p}\right)$ is equi-integrable (see [13]).

\section{Compactness properties of bounded sequences}

In the $L^{p}$ setting, if $y^{\varepsilon}$ are deformations with equi-bounded energy, the growth conditions imply that, up to subsequences, $y^{\varepsilon} \rightarrow y$ and $D_{\alpha} y^{\varepsilon} \rightarrow 0$ in $L^{p}$, hence $D_{\alpha} y=0$ and $y=y\left(x_{3}\right)$. On the contrary, in our case

$$
\delta_{\left(\frac{D_{\alpha} y^{\varepsilon}}{\varepsilon} \mid D_{3} y^{\varepsilon}\right)} \rightarrow \mu \text { weakly* in } L_{w}^{\infty} \Rightarrow \mu=\delta_{0} \otimes \nu_{x}
$$

with $\delta_{0} \in M\left(\mathbb{R}^{3 \times 2}\right)$ and $\nu_{x} \in M\left(\mathbb{R}^{3}\right)$, but now $\nu$ may depend also on $x_{1}$ and $x_{2}$. Moreover the only relevant part of the limit parametrized measure is given by the projection on the third column.

\section{The average-projection mapping and the $\Gamma$-convergence result}

The considerations above motivate the introduction of the following mapping

$$
\rho: L_{w}^{\infty}\left(\Omega ; M\left(\mathbb{R}^{3 \times 3}\right)\right) \rightarrow L_{w}^{\infty}\left((0, \ell) ; M\left(\mathbb{R}^{3}\right)\right),
$$

defined by $\rho=\pi_{\#}^{3} \circ \operatorname{Av}^{\alpha}=\operatorname{Av}^{\alpha} \circ \pi_{\#}^{3}$, where $\operatorname{Av}^{\alpha}$ denotes average with respect to the first two variables and $\pi_{\#}^{3}$ is the image measure under the projection on the third column. The commutativity of composition follows directly from the definitions. The following $\Gamma$-convergence theorem is stated with respect to the weak* convergence in $L_{w}^{\infty}\left(\omega ; M\left(\mathbb{R}^{3 \times 2}\right)\right)$ of $\rho\left(\mu^{\varepsilon}\right) \rightarrow \nu$, under which the sequences with bounded energy are relatively compact (see [13], Lemma 5.1).

Theorem 1. Let $f$ be a real extended valued continuous function which satisfies (2). Then $\Gamma\left(\rho, w^{*} L_{w}^{\infty}\left((0, \ell) ; M\left(\mathbb{R}^{3}\right)\right)\right) \lim _{\varepsilon \rightarrow 0^{+}} I_{\varepsilon}^{\infty}(\nu)=I_{S}(\nu)$ were

$$
I_{S}(\nu)=\int_{0}^{\ell}\left\langle\nu_{t}, f_{0}\right\rangle d t-\int_{0}^{\ell} \operatorname{Av}^{\alpha} g \cdot y d t
$$

if $\nu \in \mathcal{Y}^{-q, p}\left((0, \ell) ; \mathbb{R}^{3}\right)$ with $q=p s /(p+2 s)$, and holds $+\infty$ otherwise in $L_{w}^{\infty}\left((0, \ell) ; M\left(\mathbb{R}^{3}\right)\right)$. The function $y \in W^{1, p}\left((0, \ell) ; \mathbb{R}^{3}\right)$ which appears in the expression above stays for the underlying deformation of $\nu$ with boundary condition $y(0)=0$.

From our result we can recover the $\Gamma$-limit $G_{0}$ of Acerbi, Buttazzo and Percivale as follows. Let $v \in L^{p}\left((0, \ell) ; \mathbb{R}^{3}\right)$; then

$$
G_{0}(v)=\inf \left\{I_{S}(\nu): \nu \in L_{w}^{\infty}\left((0, \ell) ; M\left(\mathbb{R}^{3}\right)\right),\langle\nu, \mathrm{id}\rangle=v^{\prime}, v(0)=0\right\}
$$

with the usual convention $\inf \varnothing=+\infty$. Moreover the infimum is attained. 


\section{The energy density of the string}

The map $f_{0}: \mathbb{R}^{3} \rightarrow \overline{\mathbb{R}}$ is the unique continuous integrand satisfying the growth conditions (4) and such that the $\Gamma$-limit $I_{S}$ is the relaxation of the functional

$$
E(y)=\int_{0}^{\ell} f_{0}\left(y^{\prime}\left(x_{3}\right)\right) d x_{3}-\int_{0}^{\ell} \operatorname{Av}^{\alpha} g \cdot y d x_{3}
$$

with respect to the weak* topology in $L_{w}^{\infty}\left((0, \ell) ; M\left(\mathbb{R}^{3}\right)\right)$. Hence $f_{0}$ can be considered to be the energy density of the string.

\section{A $3 \mathrm{D}-2 \mathrm{D}$ reduction problem}

Let $\omega$ be an open bounded subset of $\mathbb{R}^{2}$ with a regular boundary, which we assume to be the reference configuration of a membrane. We want to obtain the energy of the membrane as limit, when $\varepsilon \rightarrow 0$, of the total energy of 3D hyperelastic bodies occupying the cylindrical regions $\Omega_{\varepsilon}=\omega \times(-\varepsilon / 2, \varepsilon / 2)$.

Up to the scaling factor $1 / \varepsilon$, the total energy of the $3 \mathrm{D}$ body $\Omega_{\varepsilon}$ is

$$
I_{\varepsilon}(y)=\frac{1}{\varepsilon} \int_{\Omega_{\varepsilon}} W(D y) d x-\int_{\Omega_{\varepsilon}} g^{\varepsilon}(x) \cdot y d x,
$$

where the deformation $y: \Omega_{\varepsilon} \rightarrow \mathbb{R}^{3}$ is subject to a prescribed linear boundary condition on the lateral boundary of the cylinder, that is $y(x)=B x$ on $\Gamma_{\varepsilon}=\partial \omega \times(-\varepsilon / 2, \varepsilon / 2)$, where $B: \mathbb{R}^{3} \rightarrow \mathbb{R}^{3}$ is a linear map. The energy density $W: \mathbb{R}^{3 \times 3} \rightarrow \mathbb{R}$ is continuous, generally non-convex, and satisfies a growth condition of order $p \in(1,+\infty)$, that is

$$
C\left(|F|^{p}-1\right) \leq W(F) \leq C\left(|F|^{p}+1\right) .
$$

The body force densities $g^{\varepsilon}$ are assumed to be in $L^{p^{\prime}}$, with $1 / p+1 / p^{\prime}=1$.

\section{Scaling energies and passing to a fixed domain}

The choice of a scaling factor different from $1 / \varepsilon$ in front of the $3 \mathrm{D}$ energies would lead to other limit problems; $1 / \varepsilon^{3}$, for example, corresponds to a plate model (see for instance Friesecke, James and Müller [14]).

Under the change of variable $x_{3}^{\prime}=\varepsilon x_{3}$ (still called $x_{3}$ ) the energy functionals become

$$
I_{\varepsilon}(y)=\int_{\Omega} W\left(D_{\alpha} y \mid \frac{D_{3} y}{\varepsilon}\right) d x-\int_{\Omega} g^{\varepsilon}(x) \cdot y d x
$$

where $\Omega:=\Omega_{1}$, and $y \in W^{1, p}\left(\Omega ; \mathbb{R}^{3}\right)$ has to satisfy $y(x)=B\left(x_{1}, x_{2}, \varepsilon x_{3}\right)$ on $\Gamma=\partial \omega \times(-1 / 2,1 / 2)$. Hereafter, for the sake of simplicity, we assume the scaled body force densities to be independent of $\varepsilon$. 


\subsection{Previous results and some remarks}

Le Dret and Raoult [17] proved that $\Gamma\left(L^{p}\right) \lim _{\varepsilon \rightarrow 0} I_{\varepsilon}(y)=I_{L D R}(y)$ where

$$
I_{L D R}(y)=2 \int_{\omega} Q W_{0}\left(D_{\alpha} y\right) d x_{\alpha}-\int_{\omega} \mathrm{Av}^{3} g\left(x_{\alpha}\right) \cdot y\left(x_{\alpha}\right) d x_{\alpha}
$$

if $y \in W^{1, p}\left(\Omega ; \mathbb{R}^{3}\right), y(x)=\left(x_{1}, x_{2}, 0\right)$ on $\Gamma$ and $D_{3} y=0$. Here $Q W_{0}$ denotes the quasi-convex envelope of the function

$$
W_{0}(\bar{F}):=\min \left\{W(\bar{F} \mid z): z \in \mathbb{R}^{3}\right\}, \quad \bar{F} \in \mathbb{R}^{3 \times 2}
$$

and $\mathrm{Av}^{3}$ denotes the integral mean value with respect to $x_{3}$.

As explained in the introduction, quasi-convex integrands cannot describe any microstructure. In order to overcome this difficulty, Bhattacharya and James [8] considered a 3D body characterized also by an interfacial energy which is taken to be proportional to the square of the Hessian of the deformation; in fact they consider the functionals

$$
J_{\varepsilon, \kappa}(y)=\int_{\Omega_{\varepsilon}} W(D y)+\kappa\left|D^{2} y\right|^{2} d x
$$

where the positive constant $\kappa>0$ is fixed and the limit is taken as $\varepsilon \rightarrow 0$. The introduction of the extra term leads to a $\Gamma$-limit in which no quasi-convex envelope appears. The limit energy obtained, like the three dimensional, has an interfacial energy which makes the minimization quite hard to perform.

About one year later Shu [21] has shown that if $\varepsilon$ and $\kappa(\varepsilon)$ go to 0 then the total energy considered by Bhattacharya and James $\Gamma$-converges, in a suitable topology, to the one obtained by Le Dret and Raoult.

\section{Young measures generated by gradients}

Le Dret and Raoult, in taking the $\Gamma$-limit, used the norm topology of $L^{p}$. We shall work instead within the framework of Young measures and use a topology which provides a richer description of the microstructure.

To this aim we extend the energies $I_{\varepsilon}$ to $L_{w}^{\infty}\left(\Omega ; M\left(\mathbb{R}^{3 \times 3}\right)\right)$ by setting

$$
I_{\varepsilon}^{\infty}(\mu)=\left\{\begin{array}{cl}
I_{\varepsilon}(y) & \text { if } \exists y \in W^{1, p}, y(x)=B\left(x_{1}, x_{2}, \varepsilon x_{3}\right) \text { on } \Gamma: \mu=\delta_{D y(\cdot)} \\
+\infty & \text { otherwise in } L_{w}^{\infty}\left(\Omega ; M\left(\mathbb{R}^{3 \times 3}\right)\right),
\end{array}\right.
$$

and such extension is well defined thanks to the boundary conditions.

The effective domain of the limit problem will turn out to be the space $\mathcal{Y}^{1, p}\left(\omega ; \mathbb{R}^{3}\right)$ of Young measures generated by gradients of functions in $W^{1, p}\left(\omega ; \mathbb{R}^{3}\right)$. From the characterization of Kinderlehrer and Pedregal [16] the center of mass of such a Young measure is a gradient of a function $y \in W^{1, p}$, that is $\left\langle\mu_{x}, \mathrm{id}\right\rangle=D y(x)$, and $y$ is called an underlying deformation of $\mu$. We denote by $\mathcal{Y}_{\Gamma}^{1, p}\left(\omega ; \mathbb{R}^{3}\right)$ the subspace of $\mathcal{Y}^{1, p}\left(\omega ; \mathbb{R}^{3}\right)$ whose elements have an underlying deformation satisfying the prescribed boundary condition on $\partial \omega$. 


\section{Compactness and $\boldsymbol{\Gamma}$-convergence results}

In the $L^{p}$ setting, if $y^{\varepsilon}$ are deformations bounded in energy, the growth conditions imply that, up to subsequences, $y^{\varepsilon} \rightarrow y$ and $D_{3} y^{\varepsilon} \rightarrow 0$ in $L^{p}$, hence $D_{3} y=0$ and $y=y\left(x_{1}, x_{2}\right)$. On the contrary, in our case

$$
\delta_{\left(D_{\alpha} y^{\varepsilon}, \frac{D_{3} y^{\varepsilon}}{\varepsilon}\right)} \stackrel{*}{\rightarrow} \mu \Rightarrow \mu=\nu_{x} \otimes \delta_{0}
$$

where $\nu_{x} \in M\left(\mathbb{R}^{3 \times 2}\right)$ and $\delta_{0} \in M\left(\mathbb{R}^{3}\right)$, but now $\nu$ may depend also on the variable $x_{3}$. Moreover the only relevant part of the limit parametrized measure is given by the projection on the first two columns. These facts motivates also in this case the introduction of a suitable average-projection mapping

$$
q: L_{w}^{\infty}\left(\Omega ; M\left(\mathbb{R}^{3 \times 3}\right)\right) \rightarrow L_{w}^{\infty}\left(\omega ; M\left(\mathbb{R}^{3 \times 2}\right)\right),
$$

defined by $q:=\bar{\pi}_{\#} \circ \mathrm{Av}^{3}=\mathrm{Av}^{3} \circ \bar{\pi}_{\#}$, where $\mathrm{Av}^{3}$ denotes average with respect to the third variable and $\bar{\pi}_{\#}$ is the image measure under the projection on the first two columns. The following $\Gamma$-convergence theorem is stated with respect to the weak* convergence in $L_{w}^{\infty}\left(\omega ; M\left(\mathbb{R}^{3 \times 2}\right)\right)$ of $q\left(\mu^{\varepsilon}\right) \rightarrow \nu$, under which the sequences with bounded energy are relatively compact (see [12], Theorem 5.6).

Theorem 2. Let $W$ be a real continuous function which satisfies the growth assumptions (6). Then $\Gamma\left(q, w^{*} L_{w}^{\infty}\left(\omega ; M\left(\mathbb{R}^{3 \times 2}\right)\right)\right) \lim _{\varepsilon \rightarrow 0} I_{\varepsilon}^{\infty}(\nu)=I_{M}(\nu)$ were

$$
I_{M}(\nu)=\int_{\omega}\left\langle\nu_{x_{\alpha}}, W_{0}\right\rangle d x_{\alpha}-\int_{\omega} \operatorname{Av}^{3} g \cdot y d x_{\alpha}
$$

if $\nu \in \mathcal{Y}_{\Gamma}^{1, p}\left(\omega ; \mathbb{R}^{3}\right)$ and holds $+\infty$ otherwise in $L_{w}^{\infty}\left(\omega ; M\left(\mathbb{R}^{3 \times 2}\right)\right)$. The function $y \in W^{1, p}\left(\omega ; \mathbb{R}^{3}\right)$ which appears in the expression above denotes the underlying deformation of $\nu$ which satisfies the boundary condition $y(x)=B\left(x_{1}, x_{2}, 0\right)$ on $\partial \omega$.

Given $y \in W^{1, p}\left(\Omega ; \mathbb{R}^{3}\right)$, the $\Gamma$-limit of Le Dret and Raoult at $y$ can be obtained from ours by taking the infimum of $I_{M}(\nu)$ over all $\nu \in L_{w}^{\infty}\left(\omega ; M\left(\mathbb{R}^{3 \times 2}\right)\right)$ which satisfy $\langle\nu, \mathrm{id}\rangle=D_{\alpha} y$ and $y(x)=\left(x_{1}, x_{2}, 0\right)$ on $\partial \omega$, with the usual convention inf $\varnothing=+\infty$. Moreover the infimum is attained.

\section{The energy density of the thin film}

For every matrix $B$, the $\Gamma$-limit $I_{M}$ is the Young measure relaxation of

$$
E_{W_{0}}(y)=\int_{\omega} W_{0}\left(D_{\alpha} y\right) d x_{\alpha}-\int_{\omega} \mathrm{Av}^{3} g \cdot y d x_{\alpha}, \quad y(x)=B\left(x_{1}, x_{2}, 0\right) \text { on } \partial \omega .
$$

The remarkable fact is that among all continuous integrands with $p$-growth, $W_{0}$ is the unique density such that the relaxation of the corresponding energy $E_{W_{0}}$ produces the functional $I_{M}$, for every linear map $B$. Hence $W_{0}$ can be considered to be the energy density of the thin film. 


\section{References}

1. E. Acerbi, G. Buttazzo, and D. Percivale, A variational definition of the strain energy for an elastic string, J. Elasticity, Vol. 25, pp.137-148 (1991).

2. G. Anzellotti, S. Baldo, and D. Percivale, Dimension reduction in variational problems, asymptotic development in $\Gamma$-convergence and thin structures in elasticity, Asymptot. Anal., Vol. 9, pp.61-100 (1994).

3. J.M. Ball and R.D. James, Fine phase mixtures as minimizers of energy, Arch. Rational Mech. Anal., Vol. 100, pp.13-52 (1987).

4. J.M. Ball and R.D. James, Proposed experimental tests of a theory of fine microstructure and the two well problem, Phil. Trans. R. Soc. London A., Vol. 338, pp.389-450 (1992).

5. P. Bělík and M. Luskin, A computational model for the indentation and phase transformation of a martensitic thin film, J. Mech. Phys. Solids, Vol. 50, pp.1789-1815 (2002).

6. P. Bělík and M. Luskin, A total-variation surface energy model for thin films of martensitic cristals, Interfaces Free Bound., Vol. 4, pp.71-88 (2002).

7. H. Ben Belgacem, Relaxation of singular functionals defined on Sobolev spaces, ESAIM Control Optim. Calc. Var., Vol. 5, pp.71-85 (2000)

8. K. Bhattacharya and R.D. James, A theory of thin films of martensitic materials with applications to microactuators, J. Mech. Phys. Solids, Vol. 47, pp.531-576 (1999).

9. M. Bocea and I. Fonseca, A Young measure approach to a nonlinear membrane model involving the bending moment, Proc. Roy. Soc. Edinburgh Sect. A Vol. 134 no. 5, pp.845-883 (2004).

10. A. Braides, $\Gamma$-convergence for beginners, Oxford University Press, 2002.

11. G. Dal Maso, An introduction to $\Gamma$-convergence, Birkhäuser, 1993.

12. L. Freddi and R. Paroni, The energy density of martensitic thin films via dimension reduction, Interfaces and Free Boundaries, Vol. 6, pp.439-459 (2004).

13. L. Freddi and R. Paroni, A 3D-1D Young measure theory of an elastic string. Asymptotic Analysis, Vol. 39 n.1, pp.61-89 (2004)

14. G. Friesecke, R.D. James and S. Müller, A theorem on geometric rigidity and the derivation of nonlinear plate theory from three-dimensional elasticity, Commun. Pure Appl. Math., Vol. 55, pp.1461-1506 (2002).

15. G. Friesecke, R.D. James and S. Müller, A hierarchy of plate models derived from nonlinear elasticity by $\Gamma$-convergence, Arch. Rational Mech. Anal., Vol. 180, pp.183-236 (2006).

16. D. Kinderlehrer and P. Pedregal, Characterizations of Young measures generated by gradients, Arch. Rational Mech. Anal., Vol. 115, pp.329-365 (1991).

17. H. Le Dret and A. Raoult, The nonlinear membrane model as variational limit of nonlinear three-dimensional elasticity, J. Math. Pures Appl., Vol. 74, pp.549-578 (1995).

18. M.G. Mora and S. Müller, A nonlinear model for inextensible rods as a low energy $\Gamma$-limit of three-dimensional nonlinear elasticity, Ann. I. H. Poincaré, Vol. 21, pp.271-293 (2004).

19. M.G. Mora and S. Müller, Derivation of a rod theory for multiphase materials, Calc. Var. Partial Differential Equations, Vol. 28, pp.161-178 (2007).

20. P. Pedregal, Parametrized measures and variational principles, Progress in nonlinear differential equations and their applications, Birkhäuser, 1997. 
21. Y.C. Shu, Heterogeneous thin films of martensitic materials, Arch. Rational Mech. Anal., Vol. 153, pp.39-90 (2000).

22. L.C. Young, Lectures on the calculus of variations and optimal control theory, W. B. Saunders Co., 1969. 